\title{
INFLUÊNCIA DO HÁBITO DE EXERCÍCIO NA INFÂNCIA E ADOLESCÊNCIA NA FLEXIBILIDADE DE ADULTOS SEDENTÁRIOS
}

\author{
INFLUENCE OF EXERCISE HABITS DURING CHILDHOOD AND ADOLESCENCE ON FLEXIBILITY \\ OF SEDENTARY ADULTS
}

\author{
INFLUENCIA DE LOS HÁBITOS DE EJERCICIO EN LA INFANCIA Y LA ADOLESCENCIA SOBRE \\ LA FLEXIBILIDAD DE ADULTOS SEDENTARIOS
}

Tiago de Oliveira Chaves ${ }^{1}$
(Educador Físico)
Débora Helena Balassiano ${ }^{1}$
(Médica)
Claudio Gil Soares de Araújo 1,2,3
(Médico)

1. Clínica de Medicina do Exercício CLINIMEX, Rio de Janeiro, RJ, Brasil.

2. Programa de Pós-Graduação em Cardiologia - Universidade Federal do Rio de Janeiro, UFRJ, RJ, Brasil. 3. Universidade Federal do Rio de Janeiro, Instituto do Coração Edson Saad, Rio de Janeiro, RJ, Brasil.

\section{Correspondência:}

Dr. Claudio Gil S. Araújo

Clínica de Medicina do Exercício -

CLINIMEX, Rua Siqueira Campos,

93/101. Rio de Janeiro, RJ, Brasil.

22031-071. cgaraujo@iis.com.br

\section{RESUMO}

Introdução: A flexibilidade corporal é um dos componentes da aptidão física relacionada com a saúde e desempenho físico. Esse componente tende a diminuir com o envelhecimento, sendo passível de modificação por treinamento específico; por outro lado, essas adaptações favoráveis tendem a desaparecer com destreinamento. Objetivo: Avaliar a influência do histórico de exercício físico e/ou participação desportiva competitiva na juventude sobre a flexibilidade corporal em adultos que foram pouco ativos ou sedentários nos últimos cinco anos. Métodos: Análise retrospectiva de 1.388 indivíduos avaliados entre 2012 e 2015. Após aplicação de critérios de exclusão, a amostra final incluiu 533 adultos (63,6\% homens; 20-94 anos de idade) pouco ativos ou sedentários nos últimos cinco anos. Em uma breve entrevista foram obtidos os perfis de exercício físico na infância/adolescência (PEFIA) e nos últimos cinco anos de vida. Esses perfis foram agrupados em três categorias, em função da quantidade mínima de exercício recomendado para cada idade, como: abaixo, adequado ou acima. A flexibilidade foi avaliada pelo Flexiteste e o flexíndice (FLX) foi calculado - somatório dos resultados da mobilidade passiva de cada um dos 20 movimentos articulares medidos (escala de 0 a 4) -, que foi posteriormente ajustado por idade e sexo por percentis (P-FLX) (Araújo, 2008). Resultados: Homens e mulheres adultos fisicamente inativos nos últimos cinco anos tiveram P-FLX medianos, respectivamente, de 25 e 35. Quando classificados pelo PEFIA, não foram observadas diferenças entre homens ( $P=0,23)$ e mulheres ( $P=0,10)$ no $P$-FLX. Conclusão: A flexibilidade de adultos pouco ativos ou sedentários nos últimos cinco anos, quando avaliada pelo FLX, é inferior à prevista para a idade e não é influenciada pelo PEFIA, indicando que o sedentarismo recente é prejudicial à flexibilidade global e que um histórico de mais exercício e/ou esporte na juventude não parece prevenir essa deficiência.

Descritores: estilo de vida; esportes; aptidão física; avaliação; adolescente.

\section{ABSTRACT}

Introduction: Flexibility is one of the components of performance and health-related physical fitness. This component tends to decrease with aging, but can be modified through specific physical training; on the other hand, these favorable adaptations tend to disappear with detraining. Objective: To evaluate the influence of physical exercise and/ or participation in competitive sports during childhood and adolescence on flexibility in adults who have been mostly inactive, or sedentary in the last five years. Methods: Retrospective analysis of 1,388 subjects evaluated between 2012 and 2015. After applying the exclusion criteria, the final sample comprised a total of 533 adults (63.6\% men; 20-94 years old), mostly inactive or sedentary over the last five years. The childhood and youth physical exercise profile (CYPEP) and profile of exercise in the last five years were obtained in a brief interview. These data were classified into three categories, according to the minimum recommended exercise for the specific age group, into: below, adequate and above. Flexibility was assessed by the Flexitest, and the flexindex (FLX) was calculated, as the sum total of the results of passive mobility of each of the 20 joint movements measured (scale of 0 to 4); the FLX was then adjusted by age and sex reference percentiles (P-FLX) (Araújo, 2008). Results: Men and women who had been physically inactive for the last five years showed median $P$-FLX, respectively, of 25 and 35 . Using the three CYPEP categories, there were no differences between men $(P=.23)$ and women $(P=10)$ in $P$ - FLX. Conclusion: When evaluated by the $F L X$, adults who had been mostly inactive, or sedentary in the last five years showed lower levels of flexibility compared to their age-reference values, which were not influenced by CYPEP, indicating that a recent sedentary lifestyle compromised overall flexibility, and that a more activity pattern of exercise and sports during youth is unable to prevent this deficiency.

Keywords: life style; sports; physical fitness; evaluation; adolescent.

\section{RESUMEN}

Introducción: La flexibilidad es uno de los componentes de la aptitud física relacionada con la salud y el rendimiento físico. Este componente tiende a disminuir con la edad y puede ser modificado por el entrenamiento especifico; por otra parte, estos ajustes favorables tienden a desaparecer con desentrenamiento. Objetivo: Evaluar 
la influencia de la historia de ejercicio físico y/o la participación en competencias deportivas en la juventud sobre la flexibilidad de los adultos menos activos o sedentarios en los últimos cinco años. Métodos: Análisis retrospectivo de 1.388 sujetos evaluados entre 2012 y 2015. Después de los criterios de exclusión, la muestra final fue de 533 adultos (63,6\% hombres; 20-94 años) poco activos o sedentarios en los últimos cinco años. En una breve entrevista se obtuvieron perfiles de ejercicio físico en la infancia/adolescencia (PEFIA) y en los últimos cinco años. Estos perfiles se agruparon en tres categorías en función de la cantidad mínima de ejercicio recomendado para cada edad como: abajo, adecuada o arriba. La flexibilidad se evaluó mediante Flexitest y el flexindex (FLX) fue calculado mediante la adición de los resultados de la movilidad pasiva de cada uno de los 20 movimientos articulares medidos (escala de 0 a 4), que se ajustó posteriormente para la edad y el sexo en percentiles (P-FLX) (Araújo, 2008). Resultados: Hombres y mujeres adultos físicamente inactivos en los últimos cinco años tuvieron $P$-FLX medio, respectivamente, 25 y 35. Cuando se clasificaron por PEFIA, no hubo diferencias entre hombres $(P=0,23)$ y mujeres $(P=0,10)$ en $P$-FLX. Conclusión: La flexibilidad de adultos poco activos o sedentarios en los últimos cinco años, según FLX es menor de lo esperado para la edad y no está influenciada por el PEFIA, lo que indica que el reciente estilo de vida sedentario es perjudicial para la flexibilidad general y una historia de más ejercicio y/o deporte en la juventud no parece prevenir esta deficiencia.

Descriptores: estilo de vida; deportes; aptitud física; evaluación; adolescente.

\section{INTRODUÇÃO}

A aptidão física relacionada à saúde engloba componentes aeróbicos e não-aeróbicos ${ }^{1}$. Muito embora já esteja estabelecido que a condição aeróbica seja uma excelente preditora de sobrevida em indivíduos de meia-idade, nos últimos 10 a 15 anos têm se acumulado evidências de que níveis altos de força muscular ${ }^{2-4}$ ou resultados favoráveis ${ }^{5}$ no teste de sentar e levantar, um instrumento simples de avaliação dos componentes não-aeróbicos da aptidão física ${ }^{5}$, são associados com um melhor prognóstico e, consequentemente, com uma menor mortalidade por todas as causas.

Há evidências de crianças e adolescentes fisicamente ativos possuírem uma maior probabilidade de virem a manter um estilo de vida mais ativo na vida adulta6,7. É possível que algumas das adaptações ao exercício praticado nos períodos infantil e juvenil possam ser mantidas até a vida adulta, mesmo quando o indivíduo passa a ser posteriormente pouco ativo ou sedentário. Na realidade, dados recentes mostraram que a frequência cardíaca máxima, uma resposta fisiológica saudável, tendia a ser maior nos adultos sedentários com histórico de terem sido ativos ou atletas na juventude, quando comparados a outros que não possuíam essa vivência mais intensa em exercício ou esporte ${ }^{8}$.

A flexibilidade corporal, um dos componentes não-aeróbicos da aptidão física, pode ser definida como a amplitude máxima passiva fisiológica de um dado movimento articular $^{9}$ e tende a diminuir com a idade em indivíduos dos dois sexos ${ }^{10,11}$. Diversos estudos têm indicado que uma baixa flexibilidade está associada à uma menor qualidade de vida ${ }^{12}$ e a um risco mais alto de quedas em indivíduos mais velhos ${ }^{13,14}$. Embora seja plausível supor que o sedentarismo possa ser acompanhado de níveis menores de flexibilidade corporal é desconhecido se ter sido fisicamente ativo nos primeiros anos de vida poderia contribuir, minimizar ou eliminar essa tendência à redução com o envelhecimento. Sendo assim, esse estudo objetiva avaliar se o perfil de exercício físico/esporte na infância e adolescência (PEFIA) influencia a flexibilidade corporal global de indivíduos adultos que estejam sedentários ou pouco ativos fisicamente nos últimos cinco anos.

\section{MÉTODOS}

\section{Amostra}

Foram analisados 1388 indivíduos submetidos voluntariamente a uma avaliação em uma clínica privada de Medicina do Exercício no período de janeiro de 2012 a março de 2015 . O perfil dos indivíduos mostrava ser quase todos de origem caucasiana e de padrão socioeconômico alto; por outro lado, a faixa etária e a variabilidade antropométrica foram bem amplas. A avaliação era normalmente solicitada pelo médico assistente e objetivava subsidiar a orientação de programa de exercício físico; compreendia as seguintes etapas de avaliação: clínica, cineantropométrica e fisiológica, incluindo um teste cardiopulmonar de exercício máximo.

Foram incluídos nesse estudo aqueles que, concomitantemente, preenchiam os seguintes critérios: a) mais de 20 anos de idade; b) não serem atletas; c) terem sido submetidos à avaliação cineantropométrica (que incluía a flexibilidade); d) ausência de doenças ou limitações ósteomioarticulares que pudessem interferir na avaliação da flexibilidade e e) terem fornecido informações consideradas adequadas sobre seu histórico de exercício físico na infância e/ou adolescência e mais recente, indicando ter estado pouco ativo ou sedentário (a) nos últimos cinco anos que antecederam a avaliação.

A partir destes critérios foram selecionados 533 indivíduos com idade entre 20 e 94 anos. Para uma melhor caracterização da amostra foram também obtidos dados de idade, altura e peso e calculado o índice de massa corporal (IMC) - peso (kg)/altura $\left(\mathrm{m}^{2}\right)$ - sendo considerado excesso de peso e obesidade, respectivamente, IMC superior a 25 e a $30^{15} \mathrm{~kg} / \mathrm{m}^{2}$. Em seguida, os indivíduos foram divididos por sexo - 339 homens e 194 mulheres - e agrupados em três categorias, em função do PEFIA que é recomendado para a faixa etária, como: abaixo (escores 0 e 1); adequado (escore 2) ou acima (escores 3 e 4). Uma pequena parcela dos indivíduos estudados era aparentemente saudável - 8,0\% dos homens e 10,3\% das mulheres - enquanto o diagnóstico de doença coronariana era conhecido em 29,3\% dos homens e em 14,5\% das mulheres. Os restantes $62,2 \%$ dos homens e $75,2 \%$ das mulheres possuíam alguma outra doença, incluindo obesidade, hipertensão arterial, diabetes melito, doença pulmonar obstrutiva crônica e insuficiência cardíaca. Todos os indivíduos leram e assinaram o termo de consentimento livre e esclarecido antes da avaliação. A análise retrospectiva dos dados foi previamente aprovada pelo comitê de ética em pesquisa sob o número 18/2011 e está de acordo com a legislação vigente no Brasil.

\section{Perfil de exercício físico e de esporte}

A quantidade mínima de exercícios a serem realizados para promoção de saúde em adultos é de 150 minutos de exercícios aeróbicos moderados ou 75 minutos de exercício em alta intensidade por semana. 
Além disso, devem ser realizados exercícios de fortalecimento muscular, de flexibilidade e de equilíbrio ${ }^{16}$. Já as recomendações para crianças e adolescentes são mais rigorosas, preconizando a realização de exercícios de intensidade moderada ou intensa por pelo menos uma hora diária ${ }^{17}$.

Como já descrito em estudo anterior ${ }^{8}$ e exemplificado na Tabela 1, o perfil de exercício físico e/ou esporte foi obtido em uma anamnese estruturada por um dos quatro médicos especialistas atuantes na clínica, para quatro momentos da vida: infância e adolescência; vida adulta, os últimos cinco anos, esses desdobrados nos primeiros quatro anos do período e o último ano. A pergunta inicial era se o indivíduo julgava que fazia menos ou mais exercícios e esportes do que os seus pares de idade e depois essa informação era detalhada com perguntas subsequentes. A seguir, após a apreciação do médico, essa informação era devidamente categorizada, utilizando como referência o padrão de exercício físico recomendado como mínimo para cada faixa etária, em: 0 - sedentário ou pouco ativo; 1 - algo ativo (abaixo do recomendado); 2 - ativo ou moderadamente ativo (dentro do padrão recomendado), 3 - muito ou mais ativo (acima do padrão recomendado); 4 - competitivo ou bastante ativo, com padrão regular de exercício físico compreendendo alta intensidade e/ou grande volume (intensidade vezes frequência vezes duração) e/ ou participação em esporte competitivo por federações. Comparações entre quatro médicos avaliadores evidenciaram taxas de concordância entre $95 \%$ e $99 \%$ para essas classificações. Para o presente estudo, os resultados foram agrupados em apenas três categorias, como um padrão de exercício físico em relação ao recomendado para a faixa etária menor (0 e 1), igual (2) e maior (3 e 4).

\section{Flexiteste}

O Flexiteste já foi utilizado em outros estudos publicados nessa mesma revista ${ }^{18}$. Em síntese, o Flexiteste é um método de avaliação da flexibilidade corporal que analisa a mobilidade passiva máxima de 20 movimentos nas articulações do tornozelo, joelho, quadril, "tronco", punho, cotovelo e ombro. Oito movimentos são nos membros inferiores, três no tronco e nove nos membros superiores e a numeração é feita em um sentido distal-proximal, sendo cada um dos movimentos medido em uma escala crescente e descontínua de números inteiros de 0 a $4^{9}$. A medida é feita pela execução lenta do movimento pelo avaliador até a obtenção do ponto máximo da amplitude e em seguida comparada com os mapas de avaliação para pontuação ${ }^{10}$. Um exemplo de movimento é apresentado na Figura 1. Em função da natureza da escala e pelo modo como foram propositalmente desenhados os mapas de avaliação, observa-se uma distribuição gaussiana dos resultados, com tendência central igual a 2, sendo os resultados 1 e 3 menos frequentes, e os extremos, isto é, 0 e 4, ainda mais raros 9,10 .

O Flexiteste permite avaliar flexibilidade global a partir do Flexíndice $(F L X)$ que é determinado pela soma total dos resultados dos 20 movi-

Tabela 1. Exemplos de classificação do Perfil de Exercício Físico na Infância/ Adolescência (PEFIA).

\begin{tabular}{c|c|c}
\hline Escore & Classificação & Exemplo \\
\hline 0 & $\begin{array}{c}\text { Sedentário ou muito } \\
\text { pouco ativo }\end{array}$ & $\begin{array}{r}\text { Pedaladas eventuais; educação física no colégio } \\
\text { com dispensa frequente. }\end{array}$ \\
\hline 1 & Algo ativo & $\begin{array}{r}\text { Educação física com frequência normal; futebol, } \\
\text { natação, balé e outros } \\
\text { por curto período. }\end{array}$ \\
\hline 3 & Ativo ou & $\begin{array}{r}\text { Natação, futebol, vôlei, balé, tênis e outros com } \\
\text { regular /boa assiduidade. }\end{array}$ \\
\hline 4 & Moderadamente ativo ou muito ativo & $\begin{array}{r}\text { Futebol de praia; surfe; polo-aquático; } \\
\text { e/ou atividades associadas. }\end{array}$ \\
\hline
\end{tabular}

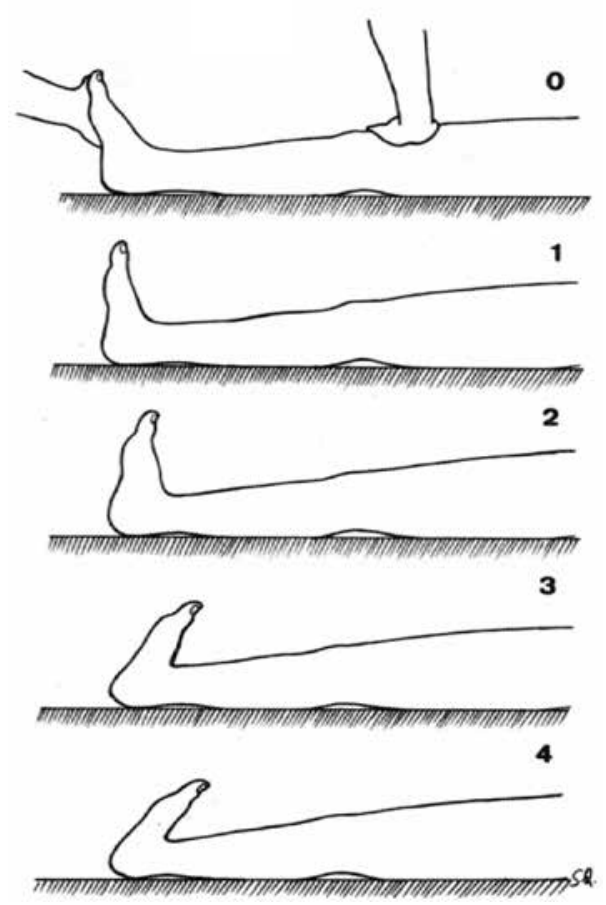

Figura 1. Mapa do Flexiteste com escores 0 a 4 para o movimento I - flexão dorsal do tornozelo.

mentos articulares. Os valores extremos máximos - 0 e 80 pontos nunca foram obtidos, assim não há os efeitos-solo e/ou teto que dificultam a utilização clínica de outros testes ${ }^{9}$. Além disso, a partir desses dados normativos pode-se determinar o percentil (P-FLX) individualizando os resultados do Flexiteste em relação à idade e sexo ${ }^{10}$, controlando assim essas duas importantes variáveis intervenientes.

Consoante com os dados de estudos anteriores ${ }^{18}$ foram utilizados todos os 20 movimentos do Flexiteste para esse estudo, tendo sido todas as avaliações realizadas por apenas três médicos com longa experiência na aplicação do método (mínimo de cinco anos). Todas as medidas de flexibilidade eram feitas uma sala com condições climáticas devidamente controladas antes da realização do teste de exercício. A sequência dos movimentos era padronizada e o indivíduo era avaliado vestindo roupas que não restringiam a mobilidade articular ${ }^{9,10}$.

\section{Análise estatística}

Foi feita uma análise descritiva para as principais variáveis do estudo. Como a distribuição dos resultados de FLX e de P-FLX não atendia critérios de normalidade (teste de Shapiro-Wilk), esses resultados foram tratados separadamente por sexo com estatística não-paramétrica e as três categorias do PEFIA comparados por ANOVA Kruskal-Wallis. Quando apropriado, o teste de qui-quadrado foi utilizado para comparar a distribuição das amostras para as variáveis nominais ou ordinais, tendo-se definido 5\% como nível de significância estatística. Todos os cálculos e figuras foram realizados no software Prism 6.01 (Graphpad, Estados Unidos).

\section{RESULTADOS}

Os resultados de idade, altura, peso e IMC para os indivíduos do sexo masculino e feminino foram (média e desvio-padrão), respectivamente: $57,6 \pm 13,8$ e 58,4 \pm 15,5 anos, $174,8 \pm 7,2$ e 159,9 $\pm 6,9 \mathrm{~cm}$, $88,3 \pm 16,1$ e $68,9 \pm 13,0 \mathrm{~kg}$ e $28,9 \pm 4,8$ e $27,0 \pm 5,1 \mathrm{~kg} / \mathrm{cm}^{2}$. Excesso de peso e obesidade ocorreram, respectivamente, em $42,5 \%$ e $37,5 \%$ dos homens e em $31,5 \%$ e $26,8 \%$ das mulheres, sendo assim mais prevalentes nos homens $(P<0,001)$. 
Em relação ao PEFIA, a distribuição dos resultados pelas três categorias foi assimétrica tanto nos homens como nas mulheres, com um maior percentual de casos na faixa dos que fizeram menos exercício do que o recomendado para a faixa etária, isso é, escores de PEFIA 0 e 1 ( $P<0,001)$. Os dados da classificação do PEFIA indicam que 49,9\% dos indivíduos tiveram pouco ou nenhuma vivência em exercício físico na infância e/ou adolescência, contrastando com apenas 18,6\% com vivência desportiva competitiva ou participação muito importante em exercício físico regular ou esporte quando jovem. Na análise por sexo temos que apenas $22,1 \%$ dos homens e $13,8 \%$ das mulheres informaram na anamnese terem sido bastante ativos ou ainda, que participaram de treinamento e competições desportivas federadas na juventude.

$\mathrm{Na}$ análise dos resultados para cada um dos 20 movimentos do Flexiteste, foram encontrados escores entre 0 a 4, confirmando o amplo espectro de flexibilidade dos indivíduos do estudo. O FLX variou entre 7 e 66, sendo, em mediana, 25 para homens (mínimo de 7 e máximo de 61) e 35 para as mulheres (mínimo de 9 e máximo de 66).

Para o P-FLX também tivemos distribuições amostrais bastante amplas, com os resultados variando de percentis 1 a 99 para ambos os sexos. Enquanto 35\% das mulheres tinham valores de P-FLX superiores a 50, isso ocorreu em apenas $25,6 \%$ dos homens incluídos no estudo, caracterizando que a flexibilidade global desses indivíduos pouco ativos ou sedentários nos últimos cinco anos tende a ser bem menor do que a esperada para a idade. Quando foram comparados os resultados do P-FLX em função do PEFIA, observou-se que não houve diferenças significativas tanto nos homens ( $P=0,232)$ como nas mulheres $(P=0,108)$. As Tabelas 2 e 3 e as Figuras 2 e 3 a seguir sumarizam os resultados das principais variáveis para as três categorias de PEFIA em homens e mulheres.

Tabela 2. Principais dados de acordo com o PEFIA para o sexo masculino ( $\mathrm{N}=339)$.

\begin{tabular}{c|c|c|c}
\hline Variável & PEFIA 0 e 1 & PEFIA 2 & PEFIA 3 e 4 \\
\hline Idade (anos) & $57,2 \pm 14,2$ & $60,6 \pm 13,6$ & $54,3 \pm 12,1$ \\
\hline Altura $(\mathrm{cm})$ & $174,8 \pm 7,2$ & $173,7 \pm 6,6$ & $176,0 \pm 7,5$ \\
\hline Peso $(\mathrm{kg})$ & $86,9 \pm 15,4$ & $87,3 \pm 17,4$ & $92,5 \pm 14,5$ \\
\hline $\begin{array}{c}\text { Índice de massa } \\
\text { corporal }(\mathrm{kg} / \mathrm{m} 2)\end{array}$ & $28,3 \pm 4,4$ & $28,8 \pm 5,3$ & $29,8 \pm 4,3$ \\
\hline PEFIA - padrăo de exercício físico na infância e adolescência &
\end{tabular}

Tabela 3. Principais dados de acordo com o PEFIA para o sexo feminino $(\mathrm{N}=194)$.

\begin{tabular}{c|c|c|c}
\hline Variável & PEFIA 0 e 1 & PEFIA 2 & PPEFIA 3 e 4 \\
\hline Idade (anos) & $60,8 \pm 15,5$ & $55,1 \pm 15,3$ & $55,2 \pm 13,1$ \\
\hline Altura (cm) & $158,9 \pm 6,8$ & $160,5 \pm 6,7$ & $162,2 \pm 6,5$ \\
\hline Peso (kg) & $69,1 \pm 13,3$ & $67,8 \pm 12,5$ & $69,3 \pm 12,3$ \\
\hline $\begin{array}{c}\text { Índice de massa } \\
\text { corporal (kg/m2) }\end{array}$ & $27,4 \pm 5,2$ & $26,3 \pm 4,8$ & $26,4 \pm 5,1$ \\
\hline
\end{tabular}

PEFIA - padrão de exercício físico na infância e adolescência

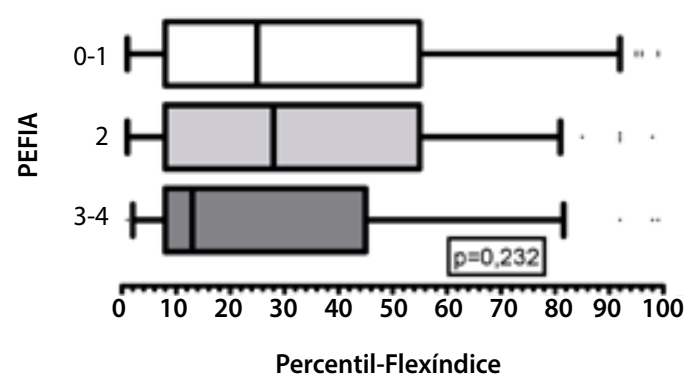

Figura 2. Percentil do Flexíndice (P-FLX) ajustado para idade e sexo de acordo com o perfil de exercício físico na infância/adolescência (PEFIA), categorizado em sedentário ou algo ativo (escores 0 e 1); mediano (escore 2) e alto ou muito alto (escores 3 e 4) ( $\mathrm{N}=339$ homens).

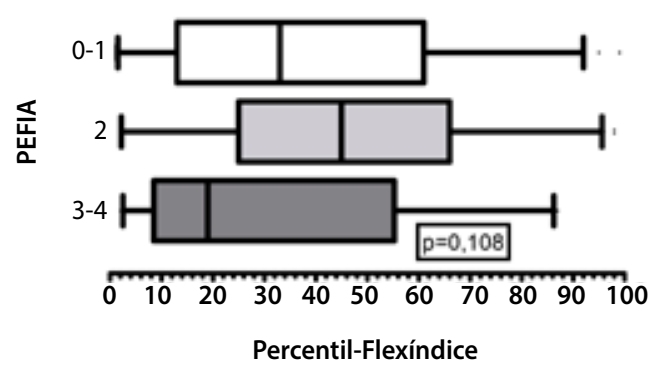

Figura 3. Percentil do Flexíndice (P-FLX) ajustado para idade e sexo de acordo com o perfil de exercício físico na infância/adolescência (PEFIA), categorizado em sedentário ou algo ativo (escores 0 e 1); mediano (escore 2) e alto ou muito alto (escores 3 e 4) ( $N=194$ mulheres).

\section{DISCUSSÃO}

O presente estudo foi realizado em uma amostra de homens e mulheres adultos não-atletas, primariamente caucasiana e de padrão socioeconômico alto, pouco ativos ou sedentários nos últimos cinco anos que antecederam à avaliação. Os resultados do IMC indicaram que uma importante parcela dos indivíduos possuía excesso de peso ou obesidade, fato comum em indivíduos com histórico recente de sedentarismo. Essas características amostrais devem ser levadas em consideração se os resultados forem extrapolados para outros grupos populacionais e representam uma relativa limitação do nosso estudo.

É apropriado também comentar a opção pelos instrumentos utilizados nesse estudo para avaliar a flexibilidade e o PEFIA. Em relação à flexibilidade, o Flexiteste é um método de avaliação da flexibilidade proposto há mais de 30 anos, tendo sido utilizado em diversos outros estudos científicos ${ }^{11,19-21}$ e que possui diversas características vantajosas, pois diferentemente de outros processos de medida da flexibilidade, este método é mais completo, pois avalia sete articulações e 20 movimentos corporais, possibilitando a obtenção de um escore global (FLX), que pode ser ajustados por idade e sexo pelo P-FLX.

Apesar de existirem outros instrumentos para a quantificação de atividade física e exercício físico, como, por exemplo, o International Physical Activity Questionnaire (IPAQ)22 e o proposto pelo American College of Sports Medicine/American Heart Association (ACSM/AHA) ${ }^{23}$, outro estudo comparando esses dois questionários mostrou que apesar de ambos serem razoavelmente reprodutíveis, os critérios da ACSM/ AHA tendem a superestimar a prática de atividade física em relação ao IPAQ ${ }^{24}$. Além disso, a utilização destes questionários é influenciada pela faixa etária, podendo não ser apropriada para indivíduos jovens e idosos 25,26 , aspectos importantes no nosso estudo. Já o PEFIA foi desenvolvido no próprio laboratório e utilizado clinicamente há diversos anos e, mais recentemente, em outras pesquisas ${ }^{8}$. Nesse sentido, a simplicidade de obtenção e de análise dos resultados do PEFIA favoreceu sobremaneira a utilização clínica e sua escolha como método de avaliação parece ter sido apropriada para o objetivo desse estudo.

Para responder o principal objetivo do estudo o delineamento ideal teria sido uma avaliação longitudinal dos indivíduos por várias décadas, algo que, contudo, não é factível de ser realizado, especialmente em uma amostra desse tamanho e com os mesmos avaliadores. Portanto, após considerar essa limitação e retornando ao principal resultado do estudo, destaca-se o fato de que diferentes níveis de PEFIA resultaram em P-FLX medianos baixos, tanto nos homens como nas mulheres. Isso claramente sugere que a atual flexibilidade global reduzida desses adultos tenha sido muito mais provavelmente influenciada negativamente pelo sedentarismo dos últimos anos do que pelo seu histórico de participação em exercício regular e/ou competições desportivas na juventude. 
A partir desses resultados fica corroborada a necessidade da prática do exercício físico regular ao longo de toda a vida e isto, provavelmente, inclui o alongamento para a obtenção de resultados apropriados na flexibilidade. Nesse sentido, há evidências de que a manutenção de níveis adequados da flexibilidade corporal pode ter impacto direto na obtenção de níveis apropriados de estabilidade postural e de equilíbrio, principalmente quando estes componentes são associados a níveis adequados de força e potência muscular ${ }^{27}$. Em adendo, sabe-se que a busca por níveis adequados nos componentes da aptidão física não-aeróbica pode contribuir para reduzir os riscos de limitações funcionais e a incapacidade física em idosos ${ }^{28}$, já que, independente do histórico de exercício físico na juventude, a flexibilidade pode ser aprimorada com treinamento específico.

Diferente dos outros componentes da aptidão física, níveis altos de flexibilidade nem sempre são associados com excelência de desempenho desportivo. Valores muitos elevados, expressos por uma hipermobilidade, podem estar associados a uma maior predisposição a lesões musculares, tendíneas e articulares e a algumas outras doenças específicas do tecido conjuntivo ou a determinadas enfermidades clínicas ${ }^{29}$. Por outro lado, quando presente em valores reduzidos pode representar um risco maior de quedas nos idosos ${ }^{14}$, diminuindo assim a qualidade de vida dos mesmos e prejudicando a execução de atividades rotineiras, tal como sentar e levantar do solo ${ }^{30}$. Dessa forma, os resultados deste estudo sugerem que, em relação à flexibilidade corporal de adultos, parece ser conveniente a inclusão desse tipo de exercício nos programas de exercício físico para que possa ser otimizado esse componente não-aeróbico da aptidão física, aparentemente, independente do histórico de sua prática na juventude.

Embora existam limitações no estudo é importante destacar também os aspectos positivos, tais como a natureza retrospectiva do estudo, o grande tamanho da amostra selecionada sob critérios de inclusão claramente estabelecidos e o fato de todos os dados terem sido obtidos por apenas três médicos especialistas em Medicina do Exercício e do Esporte e experientes nos métodos utilizados no estudo.

\section{CONCLUSÃO}

Em conclusão, o PEFIA não influencia a flexibilidade global de adultos que estejam pouco ativos ou sedentários nos últimos cinco anos. Portanto, para que a flexibilidade corporal possa ser mantida em níveis apropriados para a saúde na vida adulta, é provavelmente necessário que exercícios específicos sejam utilizados ao longo de toda a vida.

\section{AGRADECIMENTOS}

Os autores agradecem ao CNPq e FAPERJ pelo apoio dado a esta pesquisa.

Todos os autores declararam não haver qualquer potencial conflito de interesses referente a este artigo.

CONTRIBUIÇÕES DOS AUTORES: Cada autor contribuiu individual e significativamente para o desenvolvimento do manuscrito. CGSA (0000-0001-6679-6695)* e DHB $(0000-0002-2549-0013)^{*}$ participaram da coleta de dados para os resultados do estudo. TOC (0000-0002-5566-2883)* e CG avaliaram os dados da análise estatística. TOC, DHB e CGSA contribuíram com a concepção intelectual do estudo, pesquisaram a literatura, discutiram os resultados e participaram da redação do manuscrito Todos os autores contribuíram com o conceito intelectual do estudo. *Número ORCID (Open Researcher and Contributor ID).

\section{REFERÊNCIAS}

1. Araújo CG. Componentes aeróbico e não-aeróbicos da aptidão física: fatores de risco para mortalidade por todas as causas. Rev Factores Risco. 2015;35(1-3):36-42.

2. Leong DP, Teo KK, Rangarajan S, Lopez-Jaramillo P, Avezum A Jr, Orlandini A, et al. Prognostic value of grip strength: findings from the Prospective Urban Rural Epidemiology (PURE) study. Lancet. 2015;386(9990):266-73.

3. Ruiz JR, Sui X, Lobelo F, Morrow JR Jr, Jackson AW, Sjöström M, et al. Association between muscular strength and mortality in men: prospective cohort study. BMJ. 2008;337:a439.

4. Volaklis KA, Halle M, Meisinger C. Muscular strength as a strong predictor of mortality: A narrative review. Eur J Intern Med. 2015;26(5):303-10.

5. Brito LB, Ricardo DR, Araujo DS, Ramos PS, Myers J, Araujo CG. Ability to sit and rise from the floor as a predictor of all-cause mortality. Eur J Prev Cardiol. 2014;21(7):892-8.

6. Cleland V, Dwyer T, Venn A. Which domains of childhood physical activity predict physical activity in adulthood? A 20-year prospective tracking study. Br J Sports Med. 2012;46(8):595-602.

7. Huotari P, Nupponen H, Mikkelsson L, Laakso L, Kujala U. Adolescent physical fitness and activity as predictors of adulthood activity. J Sports Sci. 2011;29(11):1135-41

8. Balassiano DH, Araújo CG. Frequência cardíaca máxima: influência da experiência desportiva na infância e adolescência. Arq Bras Cardiol. 2013;100(4):333-8.

9. Araújo CG. Flexiteste: um método completo para avaliar a flexibilidade. São Paulo: Manole; 2005.

10. Araújo CG. Avaliação da flexibilidade: valores normativos do flexiteste dos 5 aos 91 anos de idade. Arq Bras Cardiol. 2008;90(4):280-7.

11. Medeiros HB, Araujo DS, Araujo CG. Age-related mobility loss is joint-specific: an analysis from 6,000 Flexitest results. Age. 2013;35(6):2399-407.

12. Coelho CW, Araújo CG. Relação entre aumento da flexibilidade e facilitações na execução de ações cotidianas em adultos participantes de programa de exercício supervisionado. Rev Bras Cineantropom Desempenho Hum. 2000;2(1):31-41.

13. Emilio EJ, Hita-Contreras F, Jimenez-Lara PM, Latorre-Roman P, Martinez-Amat A. The association of flexibility, balance, and lumbar strength with balance ability: risk of falls in older adults. J Sports Sci Med. 2014;13(2):349-57.

14. Gill TM, Pahor M, Guralnik JM, McDermott MM, King AC, Buford TW, et al. Effect of structured physical activity on prevention of serious fall injuries in adults aged 70-89: randomized clinical trial (LIFE Study). BMJ. 2016;352:i245

15. Ricardo DR, Araújo CG. Índice de massa corporal: um questionamento científico baseado em evidências. Arq Bras Cardiol. 2002;79(2):61-9.

16. Garber CE, Blissmer B, Deschenes MR, Franklin BA, Lamonte MJ, Lee IM, et al. American College of
Sports Medicine position stand. Quantity and quality of exercise for developing and maintaining cardiorespiratory, musculoskeletal, and neuromotor fitness in apparently healthy adults: guidance for prescribing exercise. Med Sci Sports Exerc. 2011;43(7):1334-59.

17. Piercy KL, Dorn JM, Fulton JE, Janz KF, Lee SM, McKinnon RA, et al. Opportunities for public health to increase physical activity among youths. Am J Public Health. 2015;105(3):421-6.

18. Araújo CG, Araújo DS. Flexiteste: utilização inapropriada de versões condensadas. Rev Bras Med Esporte. 2004;10(5):381-4.

19. Araujo CG, Chaves CP. Adult women with mitral valve prolapse are more flexible. Br J Sports Med. 2005;39(10):720-4.

20. Signorelli GR, Duarte CV, Ramos PS, Araújo CG. Melhoria da capacidade funcional excede a da condição aeróbica: dados de 144 pacientes de programa de exercício. Rev Bras Cardiol. 2012;25(4):299-308.

21. Signorelli GR, Perim RR, Santos TM, Araujo CG. A pre-season comparison of aerobic fitness and flexibility of younger and older professional soccer players. Int J Sports Med. 2012;33(11):867-72.

22. Hagströmer M, Oja P, Sjöström M. The International Physical Activity Questionnaire (IPAQ): a study of concurrent and construct validity. Public Health Nutr. 2006;9(6):755-62.

23. Haskell WL, Lee IM, Pate RR, Powell KE, Blair SN, Franklin BA, et al. Physical activity and public health: updated recommendation for adults from the American College of Sports Medicine and the American Heart Association. Med Sci Sports Exerc. 2007;39(8):1423-34

24. Moraes SA, Suzuki CS, de Freitas ICM. Comparação entre os critérios do International Physical Activity Questionnaire e do American College of Sports Medicine/American Heart Association para a classificação do padrão de atividade física em adultos. Rev Latino-Am Enfermagem. 2013;21(4):835-40.

25. BenedettiTR, Antunes PC, Rodriguez-Añez CR, Mazo GZ, Petroski EL. Reprodutibilidade e validade do Questionário Internacional de Atividade Física (IPAQ) em homens idosos. Rev Bras Med Esporte. 2007;13:11-6.

26. Guedes DP, Lopes CC, Guedes J. Reprodutibilidade e validade do Questionário Internacional de Atividade Física em adolescentes. Rev Bras Med Esporte. 2005;11(2):151-8.

27. Costa PB, Graves BS, Whitehurst M, Jacobs PL. The acute effects of different durations of static stretching on dynamic balance performance. J Strength Cond Res. 2009;23(1):141-7.

28. Paterson DH, Warburton DE. Physical activity and functional limitations in older adults: a systematic review related to Canada's Physical Activity Guidelines. Int J Behav Nutr Phys Act. 2010;7:38.

29. Scheper MC, Engelbert RH, Rameckers EA, Verbunt J, Remvig L, Juul-Kristensen B. Children with generalised joint hypermobility and musculoskeletal complaints: state of the art on diagnostics, clinical characteristics, and treatment. Biomed Res Int. 2013;2013:1-13.

30. Brito LB, Araujo DS, Araujo CG. Does flexibility influence the ability to sit and rise from the floor? Am J Phys Med Rehabil. 2013;92(3):241-7. 